\title{
Fotodegradação do Polipropileno. Um Processo Essencialmente Heterogêneo
}

\author{
Marcelo S. Rabello e J ames R. White
}

Resumo: Este trabalho analisa alguns aspectos da fotodegradação heterogênea do polipropileno (PP) e compósitos de PP com talco. Amostras moldadas por compressão e por injeção foram expostas à radiação ultravioleta em laboratório por períodos de até 48 semanas. Procedimentos sistemáticos de exposição e coleta de material para análise foram adotados visando-se minimizar as variabilidades inerentes deste estudo. As amostras foram caracterizadas por cromatografia de permeação em gel, espectroscopia de infravermelho, calorimetria diferencial de varredura, difração de raios-X, comportamento mecânico e microscopia ótica e eletrônica. As heterogeneidades na fotodegradação do PP podem estar relacionadas com três principais aspectos: (i) natureza semi-cristalina do polímero; (ii) perfil de degradação; (iii) variações estruturais em diferentes posições nos corpos de prova. As consequências da fotodegradação heterogênea foram discutidas, enfatizando-se os efeitos nas propriedades físicas.

Palavras-chave: Fotodegradação, polipropileno, envelhecimento, degradação heterogênea.

\section{Introdução}

Sob ação da radiação ultravioleta, os materiais poliméricos sofrem uma série de reações químicas oxidativas que podem causar falhas prematuras em serviço. Entre as razões para a baixa performance de polímeros degradados estão a cisão de emaranhados moleculares e cadeias atadoras, além da formação de trincas superficiais ${ }^{1,2}$. O mecanismo de fotodegradação envolve basicamente a absorção de radiação ultravioleta e subsequente reações oxidativas em processos autocatalíticos, provocando redução no peso molecular e alteração na estrutura química ${ }^{3,4}$. A durabilidade de materiais poliméricos depende não apenas da quantidade de degradação, mas também de aspectos como a espessura da camada degradada, o tamanho dos cristais e a deterioração superficial ${ }^{2,5}$.

Em muitos aspectos, a fotodegradação do polipropileno (PP) pode ser entendida como um processo heterogêneo. Em primeiro lugar as reações oxidativas geram vários grupos químicos (como éster, aldeído, carbolina e hidroperóxidos) na estrutura molecular do PP e estes são dispostos ao acaso ao longo da cadeia polimérica. Em segundo lugar, como o PP não absorve radiação ultravioleta suficiente para causar a cisão homolítica, a iniciação da fotodegradação ocorre através de impurezas (denominadas cromóforos) como resíduos de catalizadores e hidroperóxidos produzidos no processamento ${ }^{3}$. Isto resulta em micro-reatores no material onde a degradação ocorre preferencialmente ${ }^{6-8}$, com o espalhamento desta degradação dependendo de fatores como a difusão de espécies

Marcelo S. Rabello, Departamento de Engenharia de Materiais, Universidade Federal da Paraíba, Rua Aprígio Veloso, 882, Campina Grande, Paraíba. E-mail: marcelo@dca.ufpb.br; James R. White, Materials Division, Department of MMME, University of Newcastle upon Tyne, Newcastle upon Tyne, NE1 7RU, UK. 
reativas e disponibilidade de oxigênio. Algumas vezes estes centros altamente oxidados possibilitam caminhos para a falha mecânica .

Embora a heterogeneidade na química de degradação do PP esteja razoavelmente bem estabelecida, existem poucos estudos direcionados para a análise de suas consequências na estrutura e comportamento físico de polímeros. Em um polímero de micro-estrutura complexa como o polipropileno, contendo fases amorfas e cristalinas, presença de camadas estratificadas com variáveis orientações moleculares, diferentes tamanhos de esferulitos, defeitos estruturais, etc., várias outras fontes de não homogeneidade na degradação podem existir. Este trabalho apresentará algumas evidências, diretas e indiretas, da heterogeneidade na foto-oxidação do polipropileno isotático abordando aspectos físicos como a influência da estrutura semi-cristalina, o perfil de degradação e existência de variações estruturais em diferentes posições nos produtos moldados.

\section{Experimental}

A maioria das análises descritas neste trabalho foi realizada com o polipropileno isotático GXE 35, fabricado pela ICI (UK). Em alguns casos utilizou-se também um grade de PP contendo $40 \%$ em peso de talco (ICI T40H 550). Ambos são grades de uso geral para injeção e não contêm estabilizantes de ultravioleta mas contêm uma pequena quantidade de antioxidantes para evitar degradação excessiva durante o processamento.

Corpos de prova no formato típico para ensaios de tração (ASTM D638) com 3.1mm de espessura foram produzidos por injeção utilizando-se uma injetora de rosca Butler-Smith 100/60. As temperaturas do cilindro e bico de injeção foram mantidas em $200{ }^{\circ} \mathrm{Ce}$ a pressão de injeção em 107MPa. A maioria das amostras foi injetada em molde a $40^{\circ} \mathrm{C}$. O resíduo do ponto de injeção permitiu a identificação das superfícies frontal e oposta do corpo de prova, detalhe importante nos procedimentos de exposição e caracterização, conforme descrição a seguir. Alguns corpos de prova também foram produzidos por compressão utilizando-se uma temperatura de $200^{\circ} \mathrm{C}$ e uma pressão $10 \mathrm{MPa}$. Após 5 minutos de pré-aquecimento e $5 \mathrm{mi}-$ nutos sob pressão, a placa moldada foi resfriada lentamente por convecção natural na própria prensa de moldagem.
A exposição à radiação ultravioleta foi realizada em laboratório utilizando-se lâmpadas fluorescentes UVA-340 fornecidas por Q-Panel Co. Este tipo de lâmpada emite radiação ultravioleta que coincide razoavelmente bem com o espectro solar na superfície da terra ${ }^{10}$. Um dispositivo simples de exposição foi confeccionado utilizando-se duas lâmpadas de UV, conforme mostra a Figura 1. Os corpos de prova injetados foram posicionados sempre com a superfície contendo o ponto de injeção oposta à fonte de UV (Figura 2). Esta sistemática de padronização foi adotada devido às variações estruturais do polipropileno nas duas superfícies, resultante de efeitos de pressão e de fluxo durante o preenchimento do molde ${ }^{11}$. Esta variação estrutural poderia resultar em diferentes características de foto-degradação nas duas superfícies. Como também ocorre variação estrutural ao longo do comprimento de amostras injetadas ${ }^{12}$, os corpos de prova foram divididos em zonas para efeito de caracterização (Figura 2). A intensidade da radiação ultravioleta atingindo a superfície de exposição foi mantida em $2.2 \mathrm{Wm}^{-2}$ (para a faixa de comprimento de onda de 290$320 \mathrm{~nm}$ ) e medida semanalmente com um espectroradiômetro Bentham. As características de transmissão de luz UV através do pp puro e pp contendo talco estão mostradas na Figura 3.

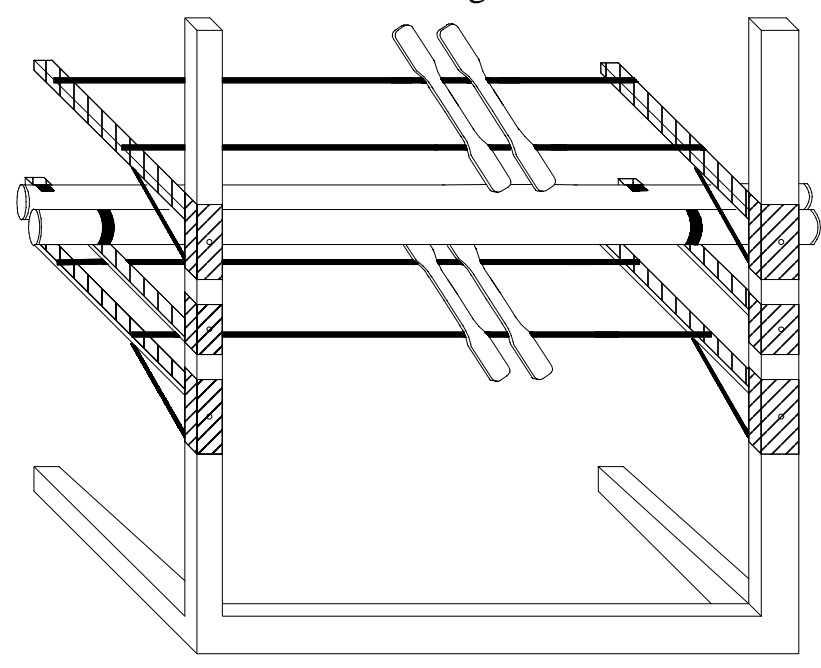

Figura 1. Dispositivo utilizado para exposição à radiação ultravioleta em laboratório, com capacidade para aproximadamente 90 corpos de prova.

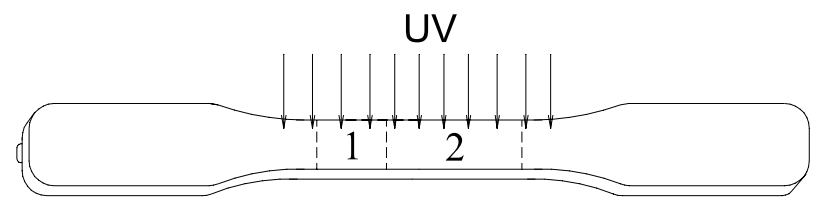

Figura 2. Posição do corpo de prova em relação à luz ultravioleta. A seção 1 foi utilizada para experimentos de difração de raios-X enquanto que a seção 2 para DSC, GPC e FTIR. 


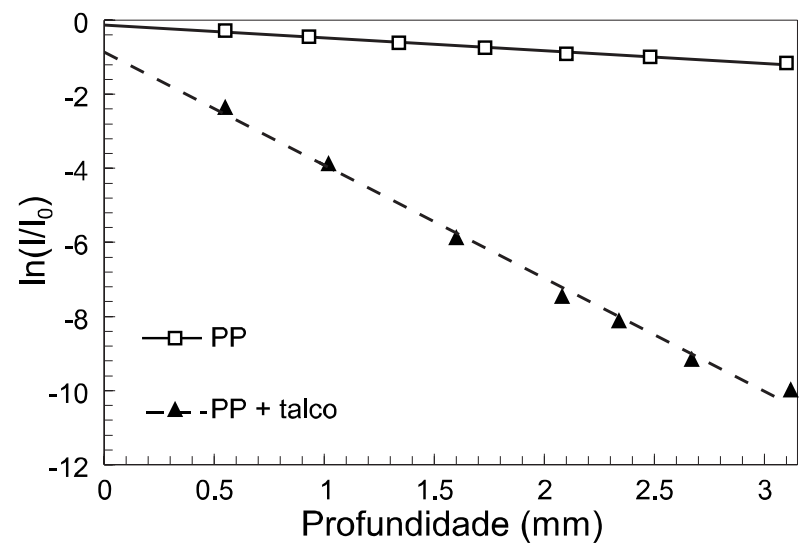

Figura 3. Variação de intensidade da radiação ultravioleta em função da profundidade no corpo de prova. Io é a intensidade na superfície e I a intensidade correspondente a cada profundidade medida. O procedimento para construção deste gráfico está descrito com detalhes em outras publicações ${ }^{13,14}$.

As amostras utilizadas para análises por calorimetria diferencial de varredura (DSC), cromatografia de permeação em gel (GPC) e espectroscopia de infravermelho com transformada de Fourier (FTIR) foram retiradas de uma camada de $0.2 \mathrm{~mm}$ de espessura da superfície exposta dos corpos de prova. Análises de FTIR foram realizadas em equipamento Nicolet $20 \mathrm{com}$ uma resolução de $4 \mathrm{~cm}^{-1}$, obtendo-se um espectro que representa a média de 32 varreduras. $\mathrm{O}$ índice de carbonila foi obtido como a razão entre as áreas sob o pico de grupos carbonila (situado na faixa $1700-1800 \mathrm{~cm}^{-1}$ ) e um pico de referência para o polipropileno (situado em $2720 \mathrm{~cm}^{-1}$ ). O pico de referência foi escolhido seguindo-se outros estudos da literatura ${ }^{15,16}$ e deve representar vibrações moleculares não afetadas pelo envelhecimento. As determinações de peso moleculares médios por GPC foram realizadas em Rapra Technology Ltd. (Shawbury, UK) cujos procedimentos aplicados para polímeros degradados foram discutidos anteriormente ${ }^{17}$. Análises térmicas por DSC foram efetuadas em célula Mettler FP85 conectada com um controlador FP90. Todas as análises foram conduzidas a uma taxa de $13^{\circ} \mathrm{C} / \mathrm{min}$, sob atmosfera de nitrogênio para evitar degradação térmica durante os ensaios. Os limites de temperatura aplicados foram: (i) aquecimento de 40 a $210^{\circ} \mathrm{C}$; (ii) resfriamento de 210 a $40^{\circ} \mathrm{C}$; (iii) aquecimento final de 40 a $210^{\circ} \mathrm{C}$.

A caracterização por difração de raios- $X$ foi realizada em difratômetro Phillips PW1730 utilizandose a radiação $\mathrm{CuK}_{\alpha}$ em amostras retiradas da posição 1 da Figura 2. Neste ensaio utilizou-se toda a amostra e não camadas superficiais para a análise; a penetração dos raios-X no polipropileno é de cerca de 0,2-0,25mm com o tipo de difratômetro utilizado ${ }^{14}$. A partir dos difratogramas obtidos (na faixa de $2 \theta=7-31^{\circ}$ ) o grau de cristalinidade foi determinado de acordo com o método desenvolvido por Weidinger \& Hermans ${ }^{18}$. Um índice de orientação cristalina foi determinado de acordo com Zipper et al. ${ }^{19}$.

Propriedades mecânicas de tração foram determinadas em máquina JJ Lloyd T5003 operando a uma velocidade de $50 \mathrm{~mm} / \mathrm{min}$. Para facilitar a comparação entre os diferentes tipos de amostras, a resistência à tração foi reportada como "resistência relativa", definida como a razão entre a resistência do material envelhecido e a do polímero virgem. Alguns corpos de prova fraturados foram analisados em microscópio eletrônico de varredura (Hitachi S-2400) após aplicação de uma cobertura de ouro. Na análise da morfologia por microscopia ótica de luz polarizada utilizou-se um Microscópio Olympus (modelo BH2) com fatias de $10-20 \mu \mathrm{m}$ microtomadas ao longo da espessura.

\section{Resultados e Discussões}

Este trabalho apresentará evidências da heterogeneidade da foto-degradação do polipropileno em termos de três principais aspectos: (i) a influência da natureza semi-cristalina; (ii) o perfil de degradação; (iii) variações estruturais. Dados experimentais pertinentes a cada tipo de efeito serão apresentados no decorrer desta seção.

\section{Influência da Natureza Semi-Cristalina}

Assim como a maioria dos polímeros cristalizáveis, a estrutura do polipropileno isotático apresenta regiões cristalinas e regiões amorfas. Devido à existência de grandes tamanhos moleculares, seções de uma mesma macromolécula estão presentes em vários cristalitos e em várias regiões não cristalinas, formando as chamadas moléculas atadoras e os emaranhados moleculares. Esta talvez seja a principal característica estrutural do PP e a presença destas moléculas atadoras confere integridade física e resistência mecânica ao produto.

A natureza de sua estrutura física e peculiaridades da cristalização do PP resulta em várias heterogeneidades em termos de fotodegradação as quais serão discutidas a seguir: (i) rejeição de grupos cromóforos e aditivos pelos cristais em crescimento; (ii) cisão preferencial de moléculas atadoras e emaranhados; (iii) diferentes taxas de difusão de oxigê- 
nio nas regiões amorfas e cristalinas; (iv) ataque nas superfícies dos cristais.

Durante a cristalização do PP impurezas presentes são geralmente rejeitadas pelos cristais em crescimento e são depositadas tanto em regiões interesferulíticas como no interior dos esferulitos ${ }^{20}$. Exemplos destas impurezas incluem moléculas de baixa isotaticidade, aditivos diversos e impurezas cromóforas. É sabido que nas proximidade de cromóforos ocorre uma taxa de foto-degradação consideravelmente maior do que em demais regiões uma vez que a foto-oxidação não é iniciada diretamente pelas moléculas de $\mathrm{PP}^{3}$. Durante o envelhecimento, o principal efeito da rejeição de cromóforos para as regiões intra- e inter-esferulíticas seria a cisão de moléculas atadoras e emaranhados, presentes na interface entre as regiões amorfas e cristalinas. A rejeição destas impurezas depende das condições de cristalização e ocorre mais facilmente em cristalização lenta ${ }^{21}$. As impurezas são rejeitadas primeiramente para a região inter-lamelar e depois para os contornos dos esferulitos. Um efeito particularmente prejudicial para o comportamento mecânico é a cisão de moléculas atadoras na região inter-esferulítica, que pode ocorrer quando existe ampla segregação de cromóforos para esta região. Vale notar que, quando presentes, aditivos estabilizantes também são rejeitados pelos esferulitos em crescimento e, portanto, atuam nos locais mais críticos, onde as reações de oxidação são de fato iniciadas.

A cisão de moléculas atadoras e emaranhados é a principal causa da grande redução nas propriedades mecânicas do PP durante exposição à radiação ultravioleta, o que ocorre antes que quantidades substanciais de produtos de degradação e danos superficiais sejam detectados. Na Figura 4 observa-se que a redução na resistência à tração do PP moldado por compressão foi consideravelmente maior do que no PP injetado. Isto ocorreu apesar da degradação química ter sido menos significativa no PP produzido por compressão (Figura 5). Acredita-se que a diferença no comportamento mecânico destes dois tipos de amostras esteja relacionado com a morfologia destes materiais. O PP injetado apresenta uma morfologia do tipo "skin-core" de camadas estratificadas com pequenos esferulitos na superfície (Figura 6a), enquanto que o PP moldado por compressão possui esferulitos consideravelmente maiores (Figura 6b). Após a compressão, a condição de cristalização empregada (resfriamento lento) provavelmente resultou em uma baixa concentração de conecções interesferulíticas e extensiva rejeição de cromóforos para os contornos dos esferulitos. Como o número de moléculas atadoras diminui com o aumento do tamanho dos esferulitos ${ }^{22}$, acredita-se que o efeito de cisão molecular nas regiões intra- e inter-esferulíticas seja mais prejudicial no PP produzido por compressão. Se a amostra possui uma pequena quantidade de moléculas atadoras, então a cisão de parte das mesmas resultará em uma grande redução nas propriedades mecânicas uma vez que as moléculas atadoras remanescentes teriam que suportar uma quantidade desproporcional de esforços mecânicos.

Um outro aspecto importante na foto-degradação de polímeros é a existência de ligações químicas tensionadas em segmentos moleculares dos emaranhados e moléculas atadoras. Dados da literatura indicam que tensões mecânicas podem acelerar a fotodegradação de polímeros ${ }^{23,24}$ devido a uma redução na barreira de energia para reação química ${ }^{25} \mathrm{ou}$

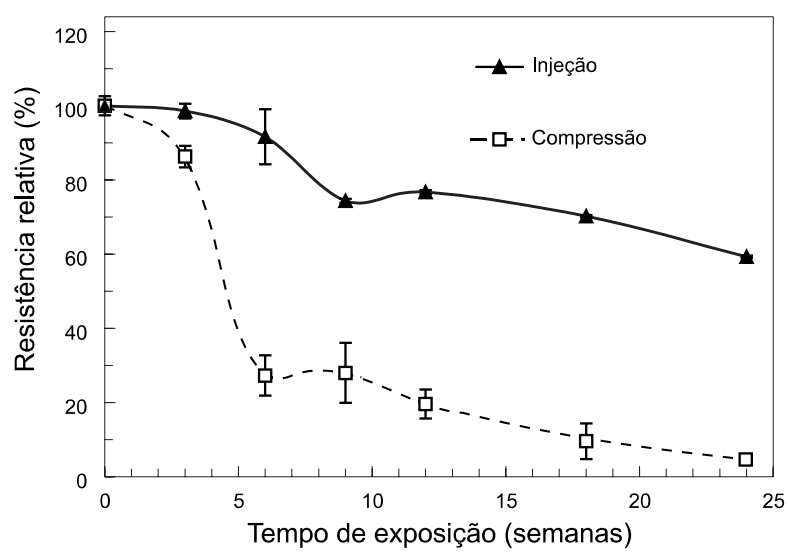

Figura 4. Efeito do tempo de exposição na resistência à tração do PP moldado por injeção e por compressão.

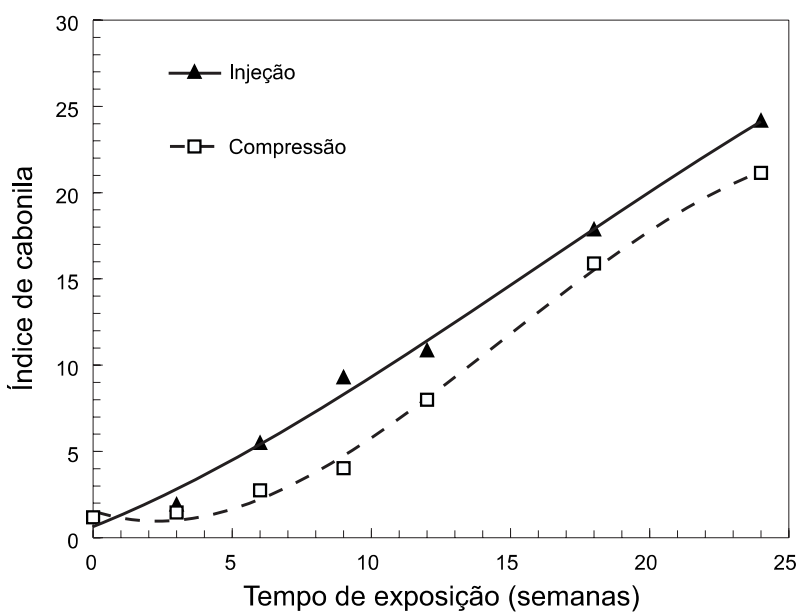

Figura 5. Índice de carbonila em função do tempo de exposição para o PP moldado por injeção e compressão. 

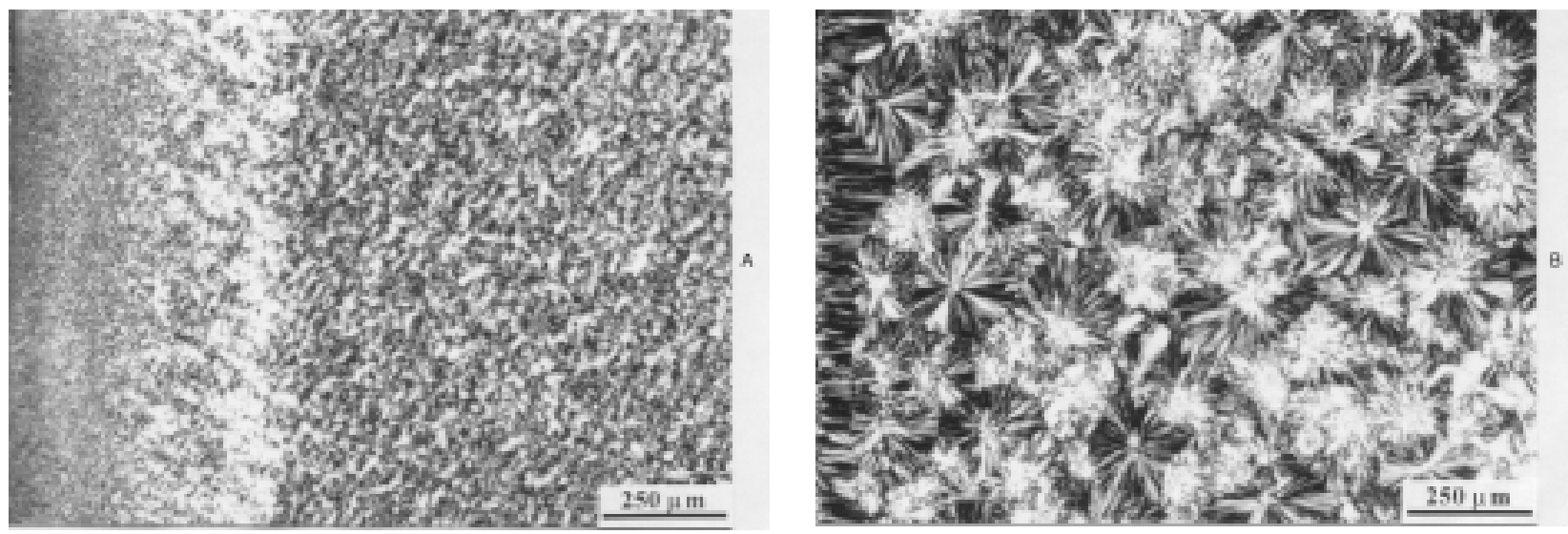

Figura 6. Microscopia ótica de luz polarizada obtida ao longo da espessura de corpos de prova moldados por injeção (a) e por compressão (b).

a uma menor taxa de recombinação dos radicais livres $^{26}$. Foi sugerido que tensões localizadas teriam um efeito similar à tensões externas na reatividade de ligações tensionadas do PP semi-cristalino e, portanto, emaranhados e moléculas atadoras seriam atacadas preferencialmente ${ }^{27}$. Este efeito também provoca heterogeneidades de degradação.

Além do efeito direto da cisão de moléculas atadoras e emaranhados nas propriedades mecânicas do polipropileno, ocorre também um outro tipo de fenômeno, chamado de quemi-cristalização. O termo significa o aumento no grau de cristalinidade do polímero durante a exposição como resultado da liberação (cisão) de segmentos moleculares nas regiões amorfas que foram impossibilitados de cristalizar durante o processamento ${ }^{28}$. Por possuírem mobilidade suficiente (a temperatura de transição vítrea do PP é menor do que a temperatura de exposição), estes segmentos liberados podem se re-arranjar em novas estruturas cristalinas, provavelmente sobre os cristais pré-existentes ${ }^{29,30}$. Um exemplo da variação da cristalinidade com o tempo de exposição está mostrado na Figura 7. Após um tempo curto de indução, a cristalinidade aumenta até atingir um valor constante, após 18-24 semanas. Conforme estudo detahado apresentado em outra publicação ${ }^{29}$, o tempo de indução foi relacionado com a redução do peso molecular enquanto que a existência de um valor limite de cristalinidade foi atribuído à crescente presença de defeitos químicos (como grupos carbonila e hidroperóxidos) nas moléculas. A principal consequência da quemi-cristalização é a formação espontânea de fissuras superficiais no produto moldado (ver Figuras 18-19 a seguir). Estas fissuras também contribuem para a redução nas propriedades mecânicas e possibilitam uma maior difusão de oxi- gênio para o interior do produto, estendendo localmente a camada degradada para posições mais profundas nas amostras (Figura 8).

$\mathrm{O}$ terceiro efeito da estrutura semi-cristalina do PP na não uniformidade da foto-degradação resulta das diferentes permeabilidades ao oxigênio através das regiões amorfas e cristalinas. Os cromóforos presentes nas regiões amorfas geram radicais livres que poderiam migrar para as regiões cristalinas, estendendo assim a degradação por todo o volume do material. Entretanto, devido ao maior empacotamento molecular, a difusão de oxigênio nos cristais de PP é extremamente baixa, resultando em uma degradação praticamente restrita à fase amorfa deste polímero ${ }^{31}$. Considerando-se apenas o efeito da morfologia na

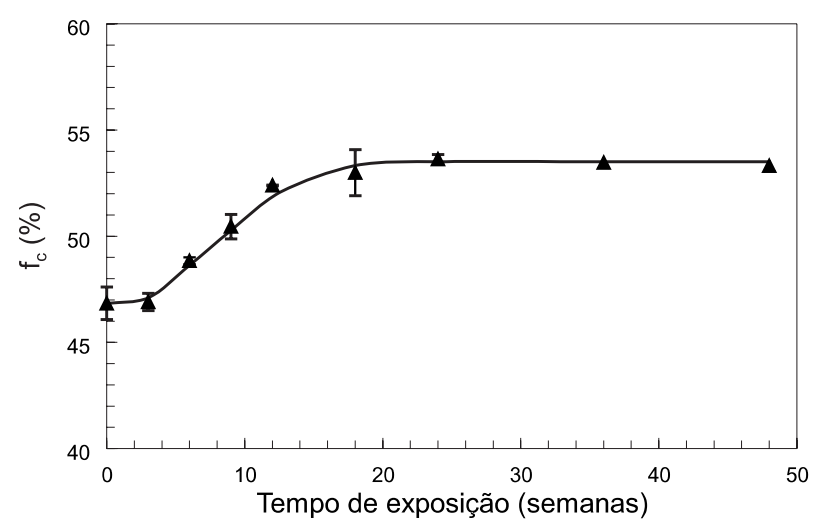

Figura 7. Variação da cristalinidade com o tempo de exposição na superfície do PP injetado.

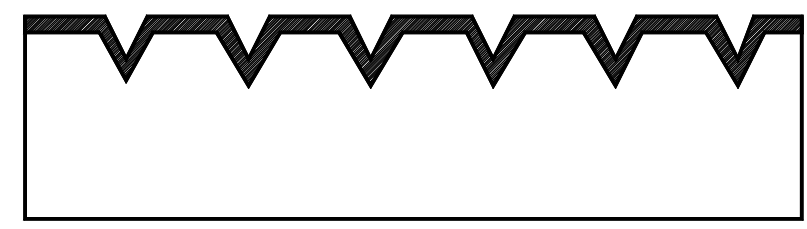

Figura 8. Representação esquemática para o efeito da presença de fissuras superficiais na camada degradada (área hachureada). 
difusão de oxigênio, espera-se que quanto mais cristalino o polímero, menor a taxa de degradação. Esta, entretanto, é uma questão controversa na literatura de degradação de polímeros ${ }^{32}$ e o oposto também tem sido reportado ${ }^{33,34}$. Por outro lado, alguns autores consideram que a extensão da fotodegradação depende apenas da concentração de grupos cromóforos e independe da sua estrutura cristalina $a^{3,4,35}$. Esta premissa pode ser verdade no caso de filmes finos, onde a degradação é controlada pela cinética de reação e não pela difusão de oxigênio. No caso de peças espessas, como as empregadas neste estudo, a reação de oxidação autocatalítica é controlada por difusão ${ }^{36}$ e, na ausência de oxigênio no local de reação, os radicais livres podem se recombinar, interrompendo as reações de propagação. Nestes casos a taxa de difusão de oxigênio deve afetar a foto-degradação. Consistente com esta abordagem, a Figura 9 mostra que a diminuição no peso molecular foi superior em amostras menos cristalinas. Estes dados foram obtidos com amostras de diferentes cristalinidades iniciais, produzidas por injeção (usando-se diferentes temperaturas de molde) e por compressão (usando-se diferentes condições de resfriamento). As temperaturas de processamento foram mantidas constantes para evitar alteração na concentração de cromóforos (como hidroperóxidos) gerados durante a moldagem.

A baixa permeabilidade de oxigênio na fase cristalina, entretanto, não evita que os cristais também sofram algumas consequências da radiação ultravioleta. Diversos autores registraram redução na cristalinidade $^{26,37}$ e alterações morfológicas ${ }^{38,39}$ devido à degradação. Estes efeitos não foram detectados neste estudo mas uma redução na temperatura de fusão cristalina foi observada (Figura 10). Devido à migração de radicais livres da região

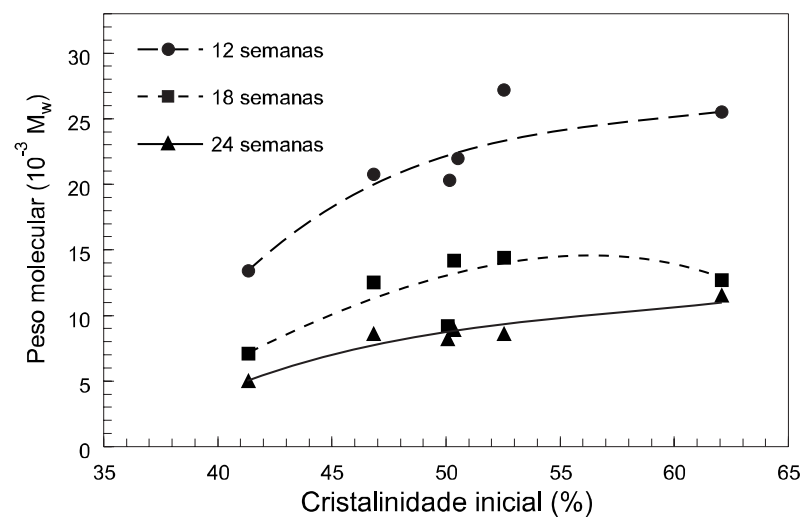

Figura 9. Efeito do grau de cristalinidade inicial no peso molecular do PP após vários tempos de exposição. amorfa (ou interfacial) para as superfícies dos cristais, houve ataque nas dobras lamelares (atente-se que os cristais de PP possuem cadeias dobradas na superfície $^{22}$ ). Isto causa um aumento na energia livre superficial dos cristais $\left(\sigma_{\mathrm{e}}\right)$, resultando em redução na temperatura de fusão ${ }^{40}$, de acordo com a conhecida equação de Hoffman \& Weeks:

$$
\mathrm{T}_{\mathrm{m}}=\mathrm{T}_{\mathrm{m}}^{0}\left(1-\frac{2 \sigma_{\mathrm{e}}}{1 \Delta \mathrm{H}}\right)
$$

onde $\mathrm{T}_{\mathrm{m}}^{\mathrm{o}}$ é a temperatura de fusão de equilíbrio, $l$ é a espessura do cristal e $\Delta \mathrm{H}$ a entalpia de fusão.

Na Figura 10 observa-se também que a redução em $\mathrm{T}_{\mathrm{m}}$ foi muito menor no PP obtido por compressão. É bastante improvável que esta diferença seja devido apenas a diferenças nas taxas de degradação (Figura 5) e, novamente, conceitos morfológicos podem explicar este comportamento. Como possui esferulitos maiores (Figura 6), o PP moldado por compressão deve possuir lamelas mais espessas do que no PP injetado e, como consequência, a contribuição das superfícies dos cristais na temperatura de fusão deve ser menor do que em amostras com cristais finos como no PP injetado.

\section{O Perfil de Degradação}

Como mencionado acima, a foto-degradação do polipropileno em produtos espessos é um processo controlado por difusão $0^{36,41}$. Isto implica em uma grande heterogeneidade de degradação ao longo da espessura dos produtos. Normalmente as camadas superficiais são mais oxidadas do que o interior do corpo de prova, conforme dados mostrados na Figura 11 para uma amostra de PP injetado. O oxigênio é consumido pelas reações oxidativas antes que consiga difundir para

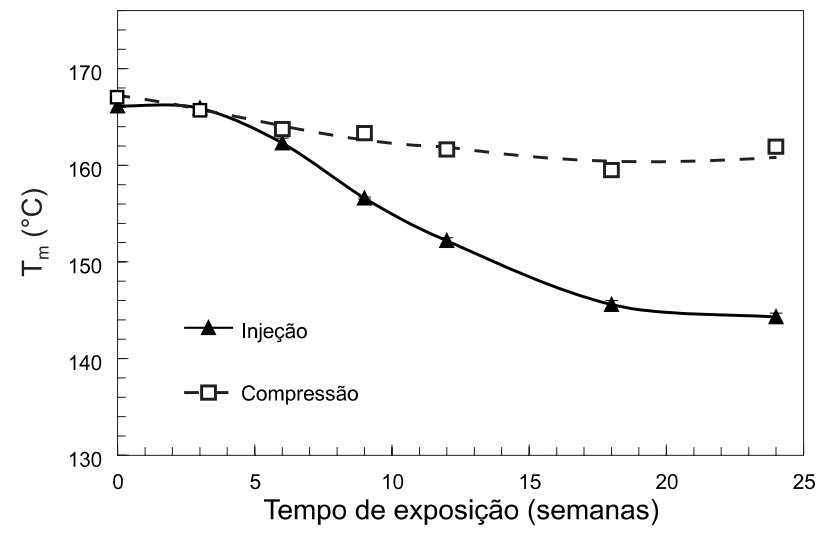

Figura 10. Temperatura de fusão do PP foto-degradado obtido por injeção e por compressão. 


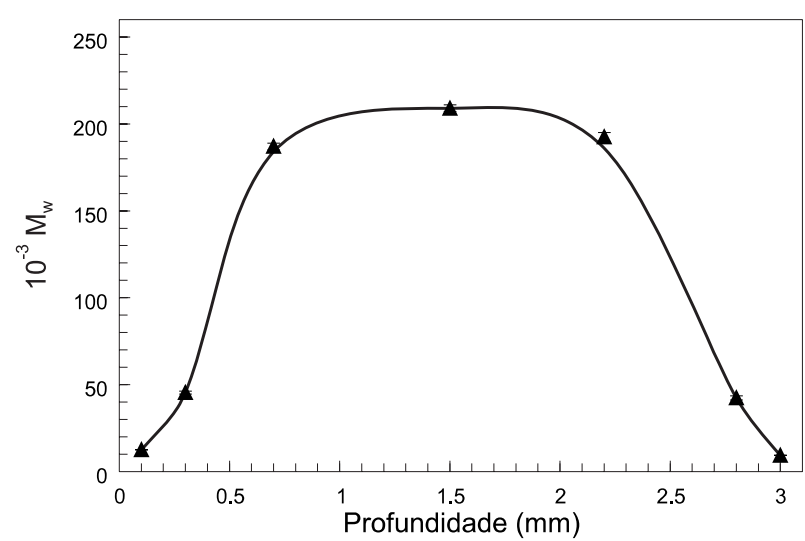

Figura 11. Variação do peso molecular ao longo da espessura de um corpo de prova injetado e exposto por 18 semanas.

o interior da amostra. Este perfil pode ser diferente no caso do envelhecimento natural uma vez que durante o período noturno (ausência de radiação UV) moléculas de oxigênio podem difundir para o interior e reagir durante o período diurno ${ }^{2}$. Caso semelhante pode ocorrer em amostras contendo estabilizantes de luz que migram para a superfície do produto. As moléculas de oxigênio não são consumidas na superfície, atingem o interior que está desprotegido de estabilizantes e, consequentemente, a superfície pode ser menos degradada do que o interior ${ }^{2}$.

Na Figura 11 observa-se que o perfil de degradação foi aproximadamente simétrico, com o peso molecular na superfície oposta à fonte de UV semelhante ao da superfície exposta. Isto ocorre porque a intensidade de luz atingindo o lado oposto da amostra é relativamente alto (Figura 3), com cerca de $50 \%$ da intensidade original. No caso do PP contendo talco, que apresenta baixa transmissão de luz, o perfil de degradação é bastante diferente, com a degradação ocorrendo apenas nas camadas superficiais da face exposta (Figura 12). Nesta figura a degradação foi avaliada pela temperatura de fusão após re-cristalização do polímero envelhecido, $\mathrm{T}_{\mathrm{m}(\mathrm{r}) \text {. }}$ Esta propriedade mostrou ser consistente com determinações de pesos moleculares ${ }^{14}$.

As características do perfil de degradação tem amplas consequências para o comportamento mecânico. O exemplo mais claro surge na comparação da resistência à tração do PP puro e PP contento talco (Figura 13). De acordo com o perfil de degradação mostrado na Figura 12, em compósitos de PP os efeitos do envelhecimento ocorreram apenas na superfície exposta, enquanto no PP puro ambas as superfícies foram degradadas. A degradação apenas na face exposta de compósitos de PP com talco foi observada também por microscopia eletrônica ${ }^{42,43}$. Como o material não

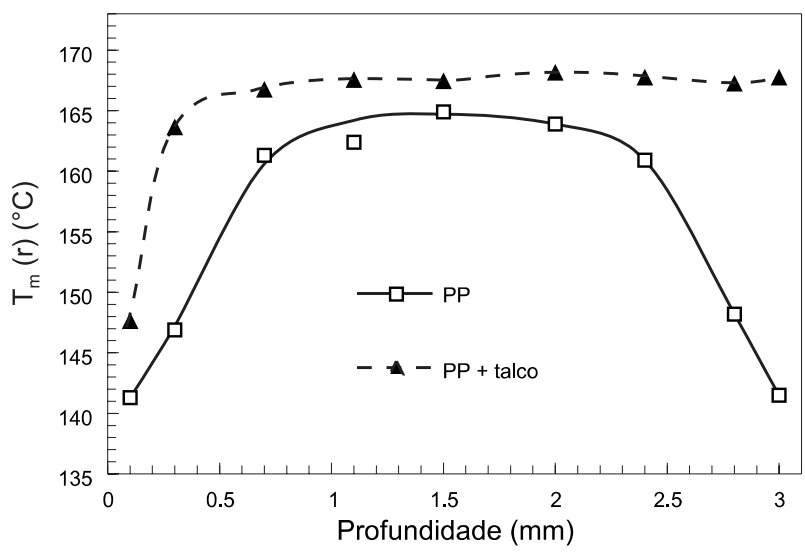

Figura 12. Perfil de degradação do PP puro e PP contendo $40 \%$ de talco. Tempo de exposição: 18 semanas.

degradado é o maior responsável em manter a integridade mecânica do produto, o desempenho de compósitos de PP foi superior ao do polímero puro.

Outro aspecto relacionado com o perfil de degradação é a recuperação parcial nas propriedades mecânicas do PP. No exemplo da Figura 14 (onde utilizou-se PP contendo $0,5 \%$ de talco como agente de nucleação $0^{44}$ ), houve uma redução brusca na resistência à tração em 6 semanas de exposição mas em 9 semanas uma recuperação parcial nesta propriedade foi observada. Este fenômeno não pode ser atribuído à dispersão de resultados pois foi verificado em muitos outros tipos de amostras de PP, produzidas sob diferentes condições ${ }^{14,32}$. Um efeito menos evidente foi mostrado na Figura 4 e Figura 13. Através de análise de superfície de fratura por microscopia eletrônica de varredura observou-se que em 6 semanas de exposição as amostras apresentaram fratura frágil, enquanto que em exposições subsequentes observouse uma banda de material dúctil entre a camada degradada e o interior (Figura 15). A explicação mais provável para este comportamento é que em amos-

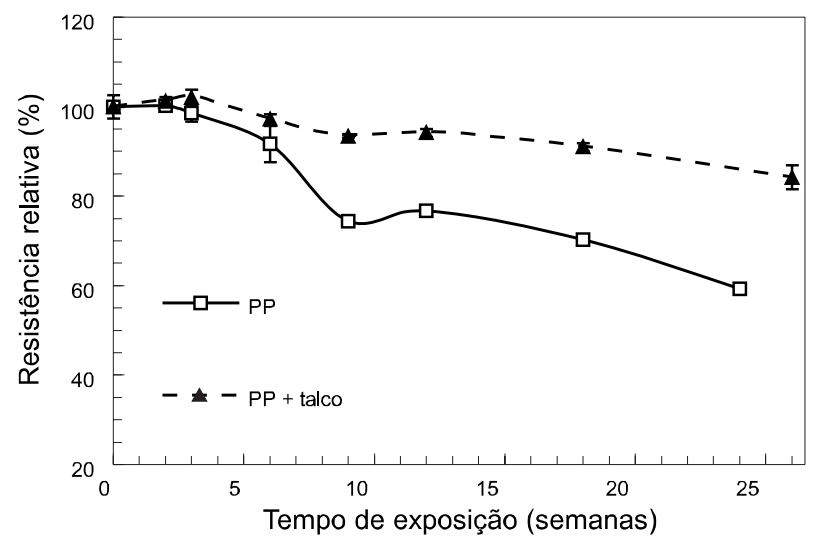

Figura 13. Efeito do tempo de exposição na resistência à tração do PP com e sem talco. 


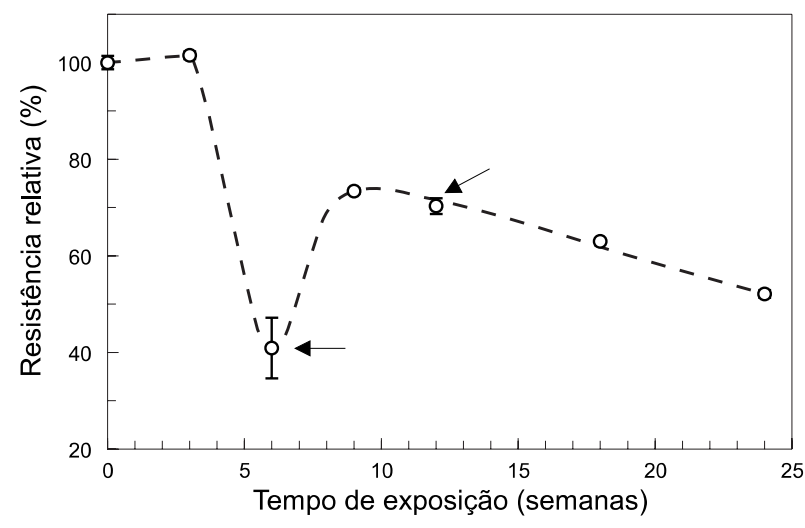

Figura 14. Resistência à tração em função do tempo de exposição do PP injetado com $0.5 \%$ de talco, utilizado como agente de nucleação ${ }^{44}$ Análises das superfícies de fratura de amostras indicadas pelas setas estão mostradas na Figura 15.

tras expostas por tempos prolongados a camada degradada apresenta-se parcialmente destacada do restante do material e, portanto, incapaz de transmitir tensões da superfície para o interior ${ }^{45}$. Este tipo de comportamento será descrito com mais detalhes em publicação futura neste periódico.

\section{Variações Estruturais}

Uma outra fonte de não uniformidade na fotodegradação do PP é a variação de estrutura que ocorre em muitos produtos. Em barras injetadas, por exemplo, o grau de cristalinidade e a orientação molecular depende da distância do canal de injeção, podendo resultar em uma dependência do nível de degradação com a posição no produto. Isto reforça a importância de se empregar procedimentos uniformes de exposição e de coleta de material para análise como os adotados neste estudo (Figura 2).

Uma grande evidência de como podem ocorrer variações dos efeitos da degradação em diferentes po-

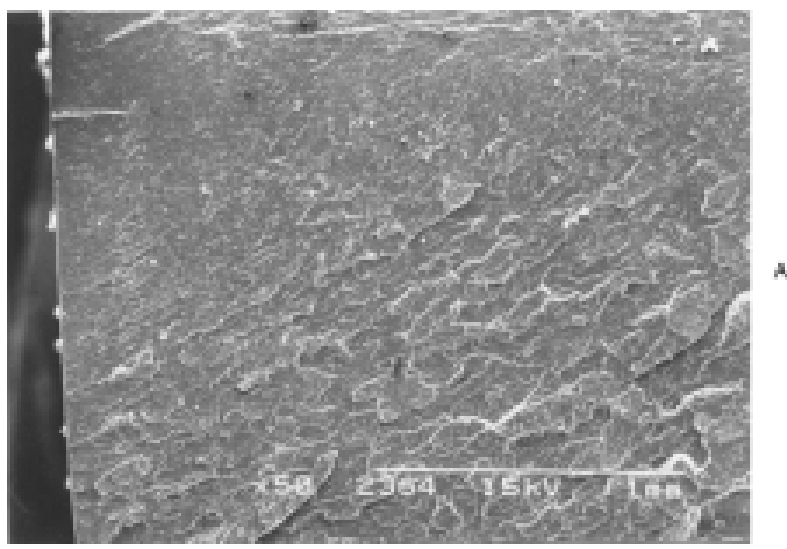

sições no corpo de prova está mostrado na Figura 16. Estas amostras possuem regiões com baixa e regiões com alta concentração de fissuras geradas durante a quemi-cristalização. Análise por difração de raios-X em diferentes posições dos corpos de prova mostraram que as regiões com baixa concentração de fissuras possuem menor orientação molecular (Figura 17).

No PP injetado as linhas de fluxo geradas durante o preenchimento do molde definem o padrão de fissuras superficiais, provavelmente porque estas são formadas paralelamente à regiões de alta orientação molecular. Por esta razão o fissuramento mostrado na Figura 16 tem a forma de arcos circulares ${ }^{46,47}$. A Figura 18 mostra as fissuras irradiando a partir do ponto de injeção de forma bastante semelhante ao material fundido entrando na cavidade do molde ${ }^{48}$. Devido à ausência de fluxo molecular durante a moldagem, o fissuramento superficial no PP moldado por compressão apresentou fissuras dispostas ao acaso (Figura 19), o que está consistente com os argumentos expostos acima. Uma análise mais detalhada do padrão de fissuras superficiais do PP foi apresentado em outro trabalho ${ }^{46}$.

A existência de uma não uniformidade de fissuramento no PP injetado coloca em dúvida a validade em se comparar o estado de deterioração superficial de diferentes amostras ou em se comparar as superfícies frontal e oposta à fonte de UV, o que é comumente encontrado na literatura ${ }^{1,49-51}$. A partir das evidências mostradas nesta seção, pode-se argumentar que a comparação em locais aleatórios das superfícies moldadas pode ser um exercício inconsistente, a menos que se esteja certo de que frequência de fissuras não varia com o local e superfícies equivalentes sejam comparadas (exemplo, ambas as superfícies contendo o ponto de injeção).

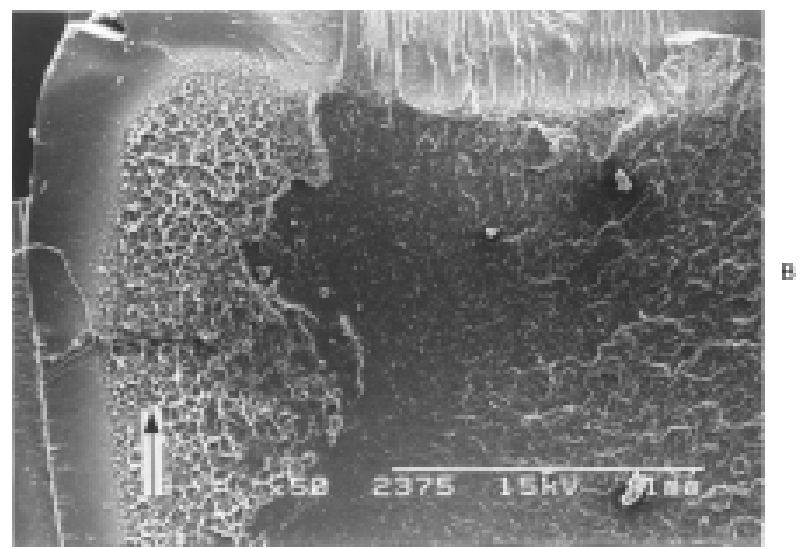

Figura 15. Microscopia eletrônica de varredura de amostras cujas propriedades estão descritas na Figura 14 . (a) 6 semanas; (b) 12 semanas de exposição. A banda de material dúctil em (b) está indicada pela seta. 


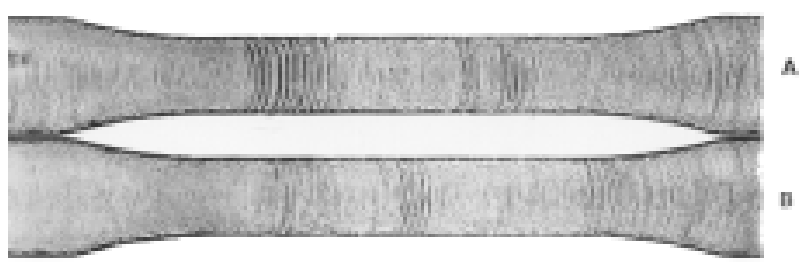

Figura 16. Distribuição de tissuras em PP injetado após 24 semanas de exposição (amostras não testadas mecanicamente). (a) superfície exposta oposta ao ponto de injeção; (b) superfície exposta contendo o ponto de injeção.
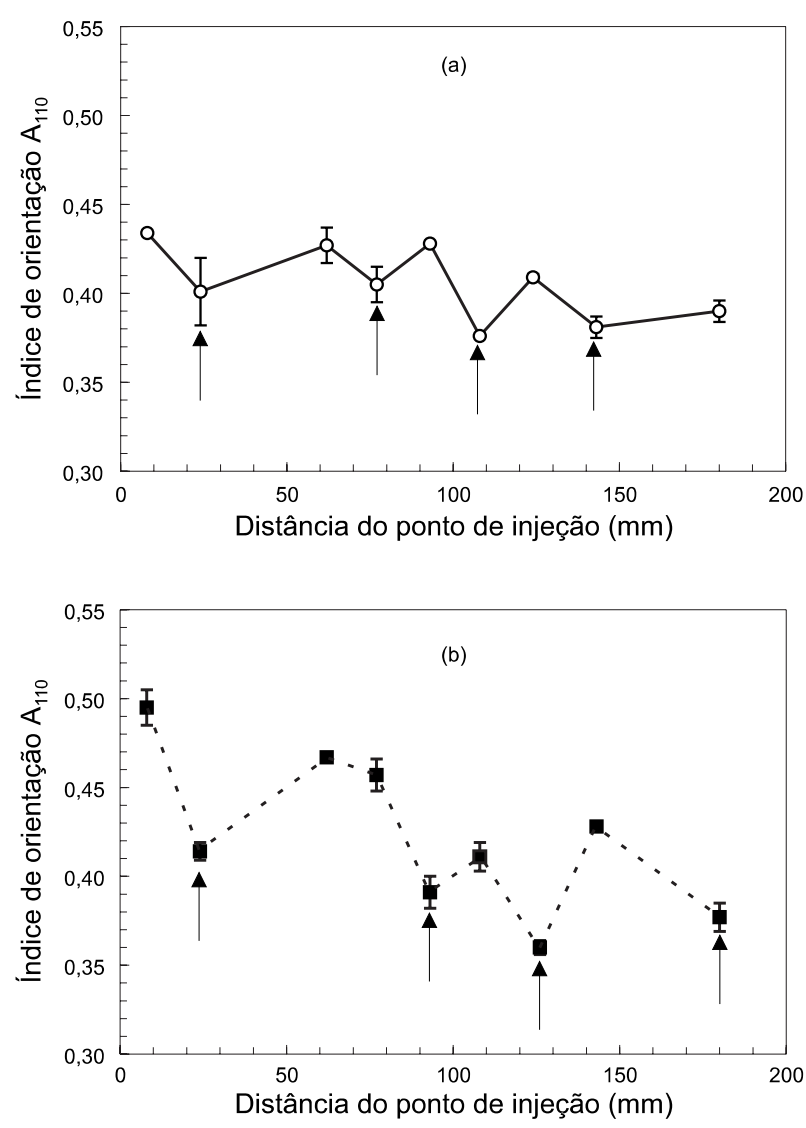

Figura 17. Variação do índice de orientação $A_{110}$ ao longo do comprimento de corpos de prova injetados. (a) superfície oposta ao ponto de injeção; (b) superfície contendo o ponto de injeção. As setas indicam as regiões com baixa frequência de fissuras na Figura 16.

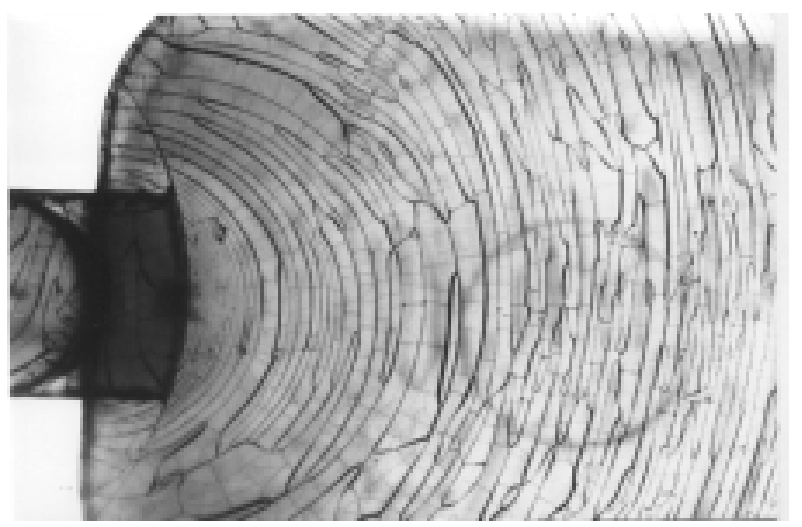

Figura 18. Fissuramento superficial na região do ponto de injeção de um corpo de prova injetado exposto por 15 semanas.
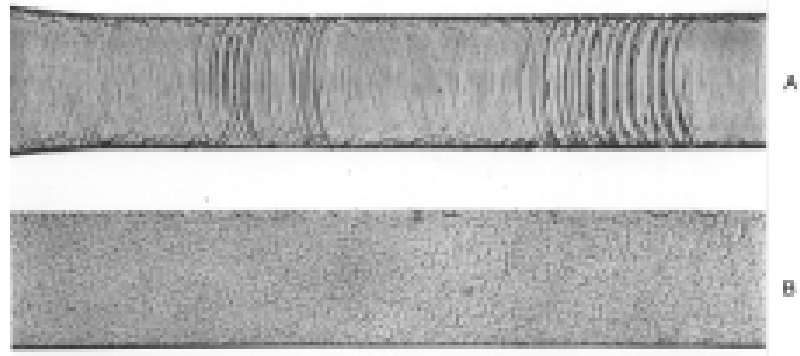

Figura 19. Fissuramento superficial no PP moldado por injeção (a) e por compressão (b), após 18 semanas de exposição.

\section{Conclusões}

As análises conduzidas neste trabalho evidenciaram várias fontes de heterogeneidade na fotodegradação do polipropileno. Como efeito direto do processo de cristalização, ocorre a rejeição de cromóforos para as regiões inter-lamelares e interesferulíticas resultando em cisão de cadeias atadoras, o que provoca perdas acentuadas nas propriedades mecânicas. Este efeito foi mais significativo em corpos de prova produzidos por compressão, que apresentam esferulitos grandes e provavelmente maior segregação de cromóforos. Outra contribuição da cisão de moléculas atadoras é o aumento da cristalinidade durante a exposição, que provoca a formação de fissuras superficiais. Embora as reações de oxidação ocorram preferencialmente nas regiões amorfas, as superfícies dos cristais são também afetadas, resultando em diminuição na temperatura de fusão.

Devido à ausência de oxigênio no interior das amostras, a fotodegradação no PP ocorre preferencialmente nas superfícies moldadas. Por outro lado, em compósitos de PP com talco apenas a superfície exposta é degradada. Esta diferença no perfil de degradação deve ser o principal fator responsável pelo melhor desempenho mecânico do PP contendo talco. Uma recuperação parcial em resistência à tração pode ocorrer, com amostras expostas por tempos prolongados apresentado desempenho superior ao de amostras expostas por tempos curtos.

Finalmente, outra importante fonte de heterogeneidade consiste em variações estruturais resultantes do processamento. Mostrou-se neste estudo que o padrão de fissuramento superficial depende da técnica de processamento e que em amostras injetadas pode haver variação na concentração de fissuras, o que está relacionada com variações de orientação na superfície. 


\section{Agradecimentos}

Os autores agradecem a ICI (UK) pelo concessão dos materiais empregados e a Rapra (UK) pelas análises de GPC. MSR é grato à Capes pela bolsa de estudos concendida.

\section{Referências Bibliográficas}

1. Schoolenberg, G.E., "A Fracture Mechanics Approach to the Effects of UV Degradation on Polypropylene", J. Mater. Sci., 23, 1580-90 (1988).

2. White, J.R.; Turnbull, A., "Weathering of Polymers: Mechanisms of Degradation and Stabilization, Testing Strategies and Modelling", J. Mater. Sci., 29, 584-613 (1994).

3. Carlsson, D.J.; Wiles, D.M., "The Photooxidative Degradation of Polypropylene. Part I. Photooxidation and Photoinitiation Processes", J. Macromol. Sci.,Rev. Macromol. Chem., C14, 65-106 (1976).

4. Rabek, J.F., "Polymer Photodegradation. Mechanisms and Experimental Methods", Chapman and Hall, London, (1995).

5. Allen, N.S., "Sunlight Induced Degradation of Polymers", Eng. Plast., 8, 247-86 (1995).

6. Margolin, A.L., "The Photooxidation of Isotactic Polypropylene as a Nonhomogeneous Process", Int. J. Polym. Mater., 24, 71-7 (1994).

7. Celina, M.; George, G.A., "Heterogeneous and Homogeneous Kinetic Analyses of the Thermal Oxidation of Polypropylene", Polym. Degrad. Stab., 50, 89-99 (1995).

8. Gugumus, F., "Thermooxidative Degradation of Polyolefins in the Solid State. Part 2: Homogeneous and Heterogeneous Aspects of Thermal Oxidation", Polym. Degrad. Stab., 52, 145-57 (1996).

9. Rapoport, N.Y.; Livanova, N.; Balogh, L.; Kelen, T., "Simulation of the durability and approach to the stabilization of polyolefins undergoing oxidative degradation under mechanical stress", Int. J. Polym. Mater., 19, 101-8 (1993).

10. Brennan, P.; Fedor, C., "43rd Annual Conference of the Composites Institute, Session 23A", SPI, Brookfield, CT 1(1988).

11. Schurz, J.M.; Zipper, P.; Lenz, J., "Structural Studies on Polymers as Prerequisites for Degradation", J. Macromol. Sci., Pure Appl. Chem., A30, 603-19 (1993).
12. Saiu, M.; Brucato, V.; Piccarolo, S.; Titomanlio, G., "Injection Moulding of iPP. An Integrated Experimental Investigation", Int. Polym. Process., 7, 267-73 (1992).

13. O'Donnell, B.; White, J.R., "Photo-Oxidation of Polystyrene under Load", J. Mater. Sci., 29, 3955-63 (1994).

14. Rabello, M.S., "The Properties and Crystallization Behaviour of Photodegraded Polypropylene" PhD Thesis, University of Newcastle upon Tyne (1996).

15. Peter, R.; Mader, E.; Ratzch, M.; Kovarova, J.; Rotschove, J.; Pospisil, J., "Charakterisierung der Stabilitat von Modifizierten Polypropylen-GlasfaseVerbunden nach Thermo- und Photooxidation", Angew. Makromol. Chem., 184, 167-81 (1991).

16. Livanova, N.M.; Zaikov, G.E., "A Scale Effect in the Durability of Oriented Narrow Polypropylene Films During Oxidation under Load. Fracture Model of Stressed Polypropylene Films", Polym. Degrad. Stab., 36, 253-9 (1992).

17. O’Donnell, B.; White, J.R.; Holding, S.R., "Molecular Weight Measurement in Weathered Polymers", J. Appl. Polym. Sci., 52, 1607-18 (1994).

18. Weidinger, A.; Hermans, P.H., "On the Determination of the Crystalline Fraction of Isotactic Polypropylene from X-Ray Diffraction”, Makromol. Chem., 50, 98115 (1961).

19. Zipper, P.; Janosi, A.; Wrentschur, E., "Scanning XRay Scattering of Mouldings from Semicrystalline Polymers", J. Phys. IV, 3, 33-6 (1993).

20. Calvert, P.D.; Ryan, T.G., "Reversible Secondary Crystallization during Cooling of Polypropylene", Polymer, 25, 921-6 (1984).

21. Keith, H.D.; Padden, F.J., "Spherulitic Crystallilzation from the Melt. I. Fractionation and Impurity Segregation and Their Influence on Crystalline Morphology", J. Appl. Phys., 35, 1270-85 (1964).

22. Bassett, D.C., "Principles of Polymer Morphology", Cambridge University Press, Cambridge, (1981).

23. Popov, A.A.; Krisyuk, B.E.; Zaikov, G.E., "The Effect of Mechanical Loads on the Low Temperature Oxidation of Polymers and the Effect of OzoneOxygenous Action on iPP”, Polym. Sci. USSR (Engl. Transl. ), 22, 1501-7 (1980).

24. Baumhardt-Neto, R.; De Paoli, M.A., "Photo-Oxidation of Polypropylene under Load", Polym. Degrad. Stab., 40, 53-8 (1993).

25. White, J.R.; Rapoport, N.Y., "Stress Effects on Polymer Durability in the Oxidative Environment", Trends Polym. Sci., 2, 197-202 (1994). 
26. Baimuratov, E.; Saidov, D.S.; Kalontarov, I.Y., "Thermal, Photo and g-Radiation Degradation of Mechanically Loaded Poly(vinyl alcohol) (PVA)", Polym. Degrad. Stab., 39, 35-9 (1993).

27. Popov, A.A.; Blinov, N.N.; Krisyuk, B.E.; Zaikov, G.E., "On The Effect of Stress on Oxidative Destruction of Polymers", Eur. Polym. J., 17, 169-73 (1981).

28. Wunderlich, B., "Macromolecular Physics. Vol. 2: Crystal Nucleation, Growth, Annealing", Academic Press, New York, (1976).

29. Rabello, M.S.; White, J.R., "Crystallization and Melting Behaviour of Photodegraded Polypropylene. 1. Chemi-Crystallization", Polymer [In Press].

30. Rabello, M.S.; White, J.R., "Chemi-Crystallization of Polypropylene under UV Irradiation" in "Antec'97", Toronto, pp 1738-42 (1997).

31. Vink, P., "The Photo-Oxidation of Polyolefins Structural and Morphological Aspects" in " Degradation and Stabilisation of Polyolefins", (ed.) N.S. Allen, Applied Science Publishers, London, pp.213-46 (1983).

32. Rabello, M.S.; White, J.R., "The Role of Physical Structure and Morphology on the Photodegradation Behaviour of Polypropylene", Polym.Degrad.Stab, 56, 55-73 (1997).

33. Dogue, I.L.J.; Mermilliod, N.; Genoud, F., "Influence of the Morphology of Polypropylene Films on the Formation and Evolution of Irradiation-Induced Peroxy-Radicals", J. Polym. Sci., Polym. Chem. Edn., 32, 2193-8 (1994).

34. Billingham, N.C.; Prentice, P.; Walker, T., "Some Effects of Morphology on Oxidation and Stabilization of Polyolefins", J.Polym.Sci.Symposium, 57, 287-97 (1976).

35. Garton, A.; Carlsson, D.J.; Wiles, D.M., "Role of Polymer Morphology in the Oxidation of Polypropylene", J. Polym. Sci., Polym. Chem. Edn., 16, 33-40 (1978).

36. Audouin, L.; Langlois, V.; Verdu, J.; De Bruijn, J.C.M., "Role of Oxygen Diffusion in Polymer Ageing: Kinetic and Mechanical Aspects”, J. Mater. Sci., 29, 569-83 (1994).

37. Kostoski, D.; Stojanovic, Z.; Gal, O.; Stannett, V., "The Effect of Gamma-Irradiation on the Morphology of Quenched Isotactic Polypropylne", Radiat. Phys. Chem., 32, 667-70 (1988).

38. Terselius, B.; Gedde, U.W.; Jansson, J.F., "Structure and Morphology of Thermally Oxidized High
Density Polyethylene Pipes", Polym. Eng. Sci., 22, 422-31 (1982).

39. Vaughan, A.S.; Stevens, G.C., "On Crystallization, Morphology and Radiation Effects in Poly(ether ether ketone)", Polymer, 36, 1531-40 (1995).

40. Zoepfl, F.; Markovic, V.; Silverman, J., "Differential Scanning Calorimetry Studies of Irradiated Polyethylene: I. Melting Temperatures and Fusion Endotherms", J. Polym. Sci., Polym. Chem. Edn., 22, 2017-32 (1984).

41. Gardette, J., "Heterogeneous Photooxidation of Solid Polymers", Angew. Makromol. Chem., 232, 85-103 (1995).

42. Rabello, M.S.; White, J.R., "Photodegradation of TalcFilled Polypropylene", Polym. Comp., 17, 691-704 (1996).

43. Chinelatto, M.A.; Agnelli, J.A.M., "Effect of the Chemical Stabilization System on the Photooxidation of Polypropylene Containing Talc and Carbon Black", Polym. Degrad. Stab., 50, 13-9 (1995).

44. Rabello, M.S.; White, J.R., "Photodegradation of Polypropylene Containing a Nucleating Agent", J.Appl.Polym.Sci. [In Press]

45. Rabello, M.S.; White, J.R., "Weatherability of Polypropylene Containing Talc Nucleating Agent" in "Antec'97", Toronto, pp 2991-5 (1997).

46. Rabello, M.S.; White, J.R., "Photodegradation of Polypropylene Mouldings Containing Weld Lines: Mechanical Properties and Surface Cracking", Plast. Rubb. Compos. Proc. Appl., 25, 237-48 (1996).

47. Rabello, M.S.; White, J.R., "Surface Cracking in Photodegraded Polypropylene" in "VII International Macromolecular Colloquium”, Gramado (RS) 31 (1996).

48. White, J.L.; Dee, H.B., "Flow Visualization for Injection Moulding of Polyethylene and Polystyrene Melts", Polym. Eng. Sci., 14, 212-22 (1974).

49. Sherman, E.S.; Ram, A.; Kenig, S., "Tensile Failure of Weathered Polycarbonate", Polym. Eng. Sci., 22, 457-65 (1982).

50. Qayyum, M.M.; White, J.R., "Plastic Fracture in Weathered Polymers", Polymer, 28, 469-76 (1987).

51. Hulme, A.; Mills, N.J., "The Analysis of Weathering Tests on Industrial Helmets Moulded from Coloured Polyethylene", Plast. Rubb. Compos. Proc. Appl., 22, 285-303 (1994). 